\title{
Resilient intracrystalline occlusions: a solid-state NMR view of local structure as it tunes bulk lattice properties
}

Ira Ben Shir ${ }^{\dagger}$, Shifi Kababya ${ }^{\dagger}$, David B. Zax ${ }^{\ddagger}$ and Asher Schmidt ${ }^{\dagger, *}$

${ }^{\dagger}$ Schulich Faculty of Chemistry and Russell Berrie Nanotechnology Institute, Technion Israel Institute of Technology, Technion City, Haifa 32000, Israel

${ }^{\ddagger}$ Department of Chemistry \& Chemical Biology, Baker Laboratory, Cornell University, Ithaca, NY 14853 USA

* Tel: 972-4-8292583

Fax: 972-4-8295703

e-mail: asher@ch.technion.ac.il 


\section{Supporting Information}

${ }^{13} \mathrm{C}$ and ${ }^{15} \mathrm{~N}$ DE MAS and ${ }^{1} \mathrm{H}\left\{{ }^{13} \mathrm{C}\right\}$ 2D-HETCOR spectra of the $\left[10 \%-{ }^{13} \mathrm{C}\right] \mathrm{CaCO}_{3}-\mathrm{Asp}$ coprecipitates, SEM images of the vaterite and calcite coprecipitates, REDOR association number simulations $\left({ }^{15} \mathrm{~N} \cdots{ }^{13} \mathrm{C} n\right)$, assumptions made for the computer model of randomly doped cubic lattice.

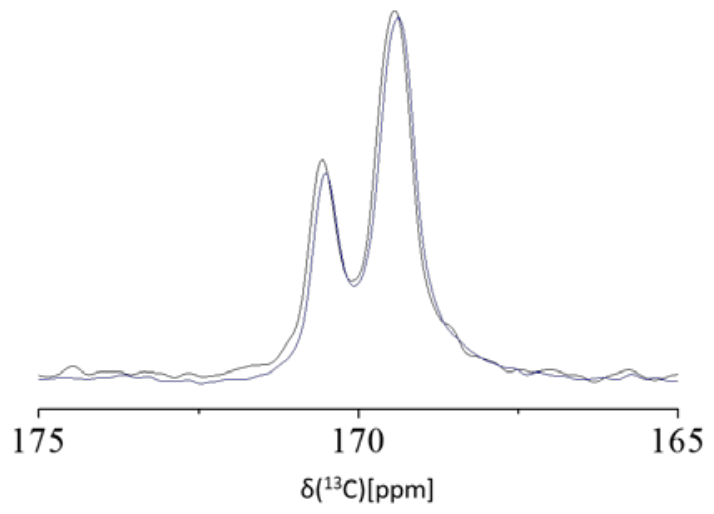

Figure S1: 75.6 MHz ${ }^{13} \mathrm{C}$ DE MAS spectra, carbonate region, of the Low-Asp (black) and High-Asp (blue) $\left[10 \%-{ }^{13} \mathrm{C}\right] \mathrm{CaCO}_{3}$-Asp coprecipitates $\left(v_{\mathrm{R}}=5,000 \mathrm{~Hz}\right.$; $\left.\mathrm{rd}=2400 \mathrm{~s}\right)$. These superimposed spectra show that vaterite is formed, independent of Asp concentration in solution (10-fold) and Asp occlusion level (2-fold).

High-[Asp] vaterite
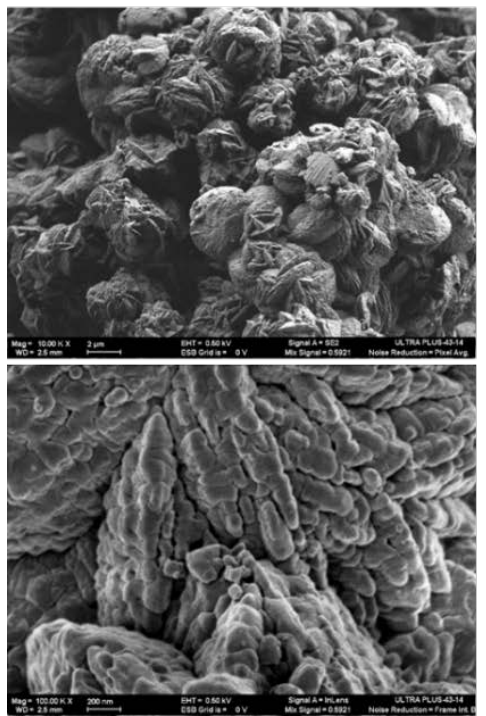

Low-[Asp] vaterite
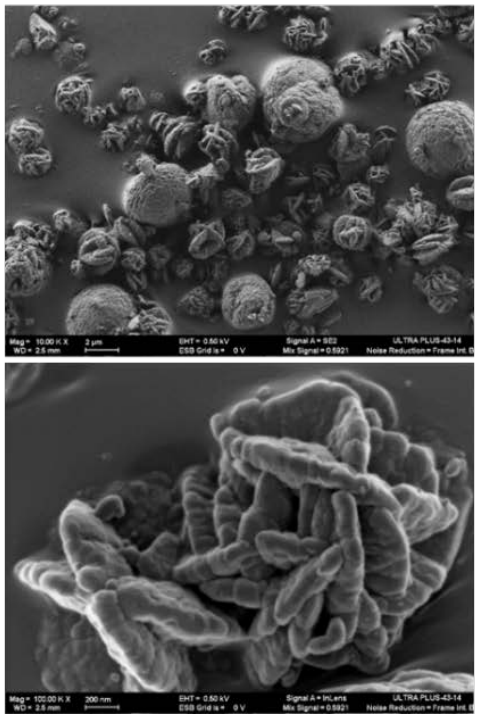

Low-[Asp] calcite
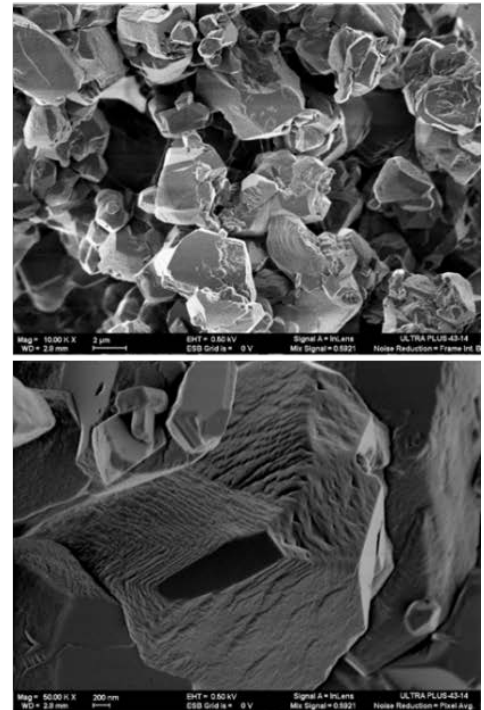

Figure S2: SEM images of the high- and low-[Asp] vaterite, and low-[asp] calcite coprecipitates. Both low- and high-[Asp] vaterite precipitates similarly exhibit $\sim 150$ $\mathrm{nm}$ nanospheres, aggregated and partially coalesced in $\sim 2 \mu \mathrm{m}$ particles. These images demonstrate that neither the morphology nor particle size of vaterite depend on Asp 
concentration in solution. Significant morphological changes are observed after conversion of low-[Asp] vaterite to calcite, where the $\sim 2 \mu \mathrm{m}$ particles display rectangular faceted edges with some remaining patterning of the original $150 \mathrm{~nm}$ nanoparticles.

A high-resolution scanning electron microscope (HR-SEM, ULTRA Plus, Zeiss, Oberkochen, Germany) was used for characterization of crystal shape and morphology of the calcium carbonates powders. The samples were carbon coated. Images were obtained in high vacuum mode at $0.5 \mathrm{kV}$. 

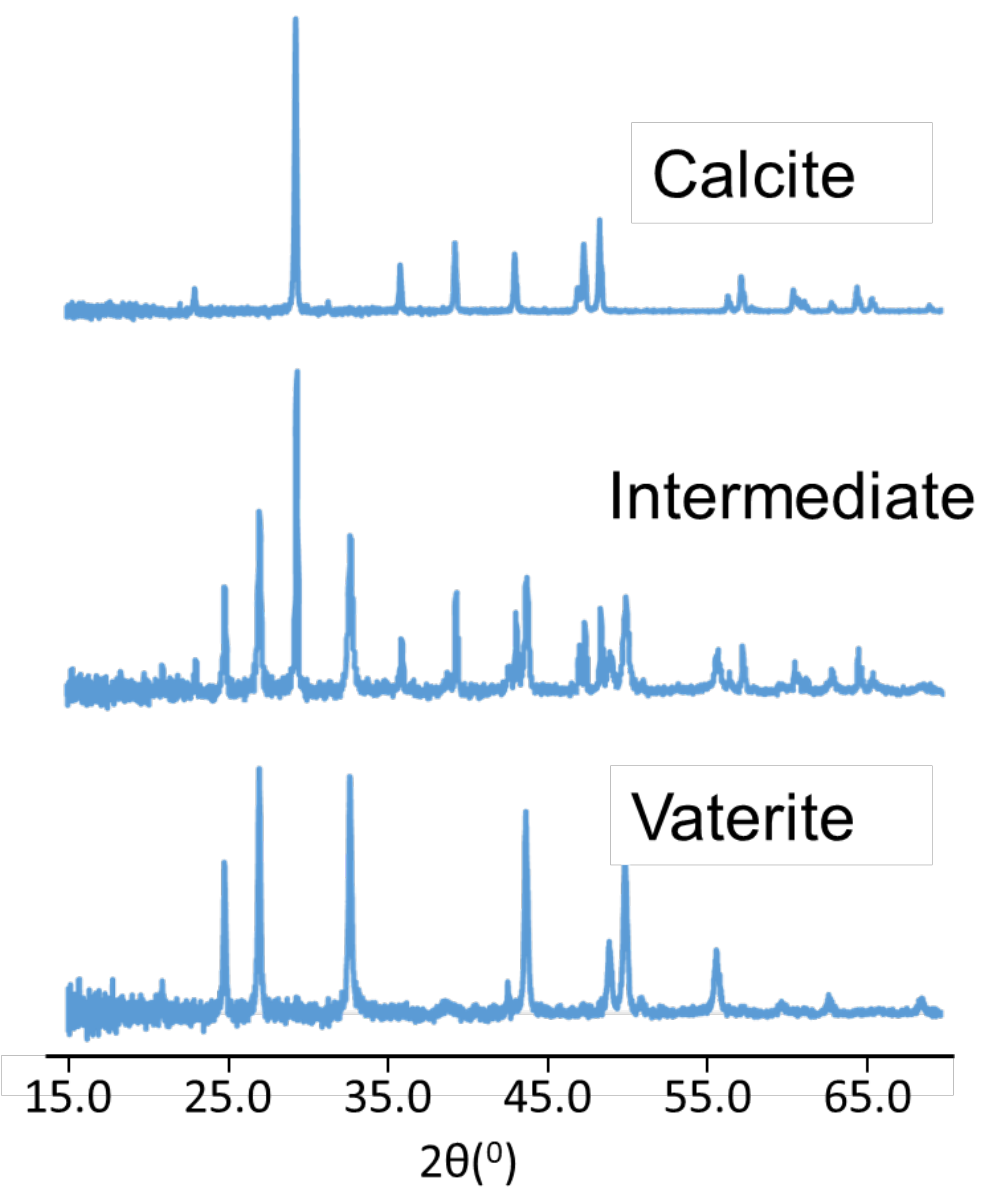

Figure S3: X-ray powder diffraction of the three coprecipitates: high-[Asp] vaterite, low-[Asp] at intermediate stage showing both vaterite and calcite, and fully transformed low-[Asp] calcite. 


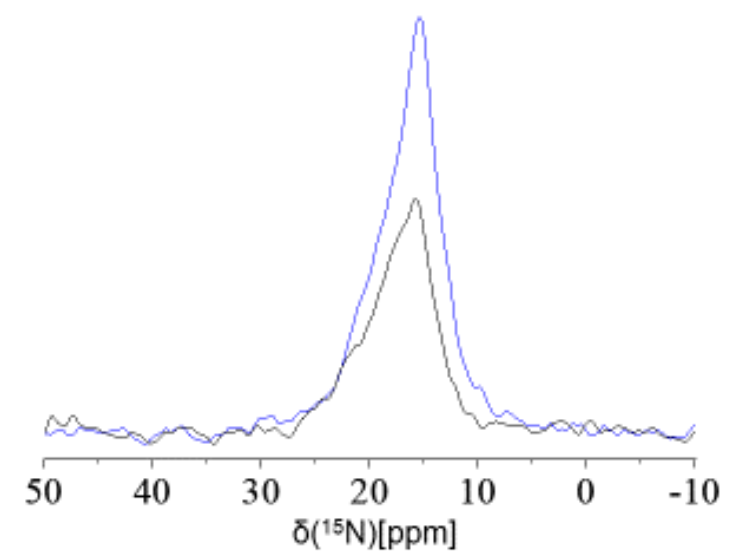

Figure S4a: $50.2 \mathrm{MHz}{ }^{15} \mathrm{~N}$ DE MAS spectra of a) Low-[Asp] (black) and High-[Asp] (blue) $\mathrm{CaCO}_{3}$-Asp coprecipitate acquired under the same experimental conditions. The spectra are drawn normalized by sample weight and number of scans so that the peak areas represent the occlusion levels. In the High-[Asp] coprecipitates Asp abundance is about 2-fold higher than in the Low-[Asp] coprecipitate, in close agreement with the amino acid analysis data.

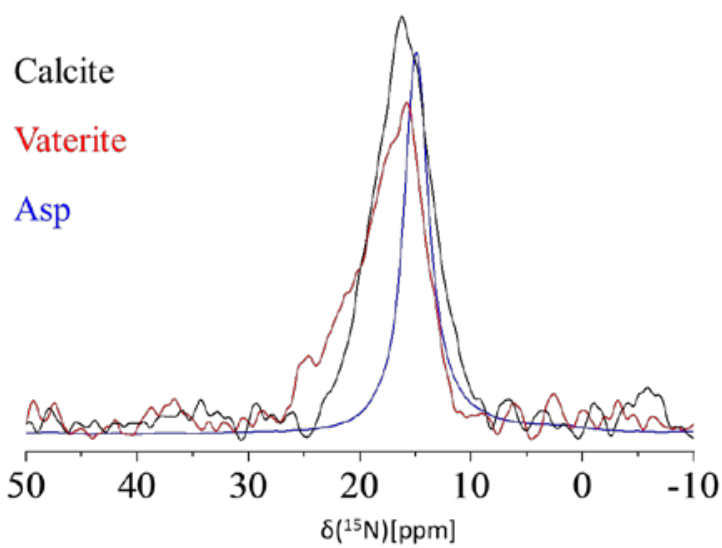

Figure S4b: $50.2 \mathrm{MHz}{ }^{15} \mathrm{~N}$ DE MAS spectra of low-[Asp] vaterite (red) and the evolved low-[Asp] calcite (black) $\mathrm{CaCO}_{3}$-Asp coprecipitate. The overlaid spectra show the changing lineshapes that attest for changes imparted to the amine chemical environments as the host lattice and the interaction shell carbonates spontaneously transform from vaterite to calcite. The ${ }^{15} \mathrm{~N}$-amine broad and asymmetric lineshapes in the calcite and vaterite samples represent heterogeneous chemical-structural environments found for Asp in the occlusion sites; the narrower linewidth seen in the calcite sample indicates that the solid phase transformation to calcite reduced disorder in the occlusion shell.

$v_{\mathrm{R}}=5,000 \mathrm{~Hz}$; rd = $10 \mathrm{~s}$; Stable isotope enrichment: Carbonates $10.0 \%-{ }^{13} \mathrm{C}$ and L-Asp $99.8 \%-{ }^{15} \mathrm{~N}$. Relaxation delay of $10 \mathrm{~s}$ yielded quantitative ${ }^{15} \mathrm{~N}$ spectra. 


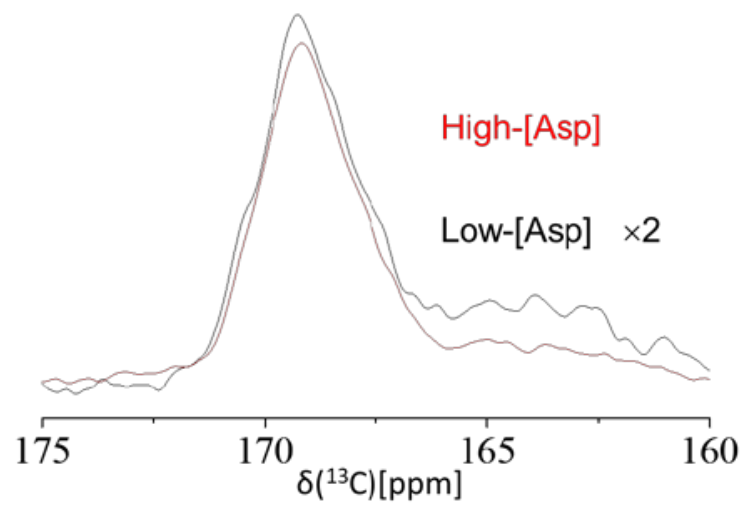

Figure S5: $75.4 \mathrm{MHz}{ }^{13} \mathrm{C}$ CP MAS spectra, carbonate region, of the transient Low[Asp] (black) and the stable High-[Asp] (red) vaterite coprecipitates. The spectra were normalized per sample weight and number of scans. The similarity of the interfacial carbonates peaks of high- or low-[Asp] indicate that the resulting interfacial carbonates are independent of Asp concentration in the precipitation solution (10-fold) and of Asp occlusion level ( 2-fold) in vaterite. Quantitatively, the doubled intensity seen in the high-[Asp] carbonate peak suggests twice as many interfacial carbonates, consistent with the 2 -fold increased occlusion level as long as Asp is found as isolated molecules, and not phase segregated or significantly aggregated.

$\left(v_{\mathrm{R}}=5,000 \mathrm{~Hz}\right.$; rd $\left.=3 \mathrm{~s}\right)$ Stable isotope enrichment: Carbonates $10.0 \%-{ }^{13} \mathrm{C}$ and L-Asp $99.8 \%{ }^{-15} \mathrm{~N}$. 


\section{${ }^{1} \mathbf{H}\left\{{ }^{13} \mathrm{C}\right\}$ 2D-HETCOR}

A further characterization of the proximity effect is provided by a modification of the classic CP MAS experiment where we probe the CP signal after a period during which ${ }^{1} \mathrm{H}$ chemical shifts dominate the evolution. The resulting experiment is known as ${ }^{1} \mathrm{H}-{ }^{13} \mathrm{C}$ 2D-HETCOR MAS NMR. CPMAS represents all ${ }^{13} \mathrm{C}$ environments close to any ${ }^{1} \mathrm{H}$ site; in contrast, this modified CPMAS associates a specific ${ }^{1} \mathrm{H}$ chemical environment, or set thereof, with the ${ }^{13} \mathrm{C}$ sites polarized by $\mathrm{CP}$ - so that crosspeaks in the $2 \mathrm{D}$ spectrum identify ${ }^{1} \mathrm{H}$ and ${ }^{13} \mathrm{C}$ chemical environments which are physically close in space differentiating between interfacial carbon sites based on their neighboring ${ }^{1} \mathrm{H}$ chemical shifts (Figure 2c).

In all coprecipitates, whether vateritic or calcitic, we identify the ${ }^{1} \mathrm{H}$ chemical environments associated with the chemical shifts of the $\mathrm{H}_{\alpha}$ and $\mathrm{H}_{\beta}\left(\mathrm{CH}\right.$ and $\left.\mathrm{CH}_{2}\right)$ groups of aspartic acid (at $\sim 3 \mathrm{ppm}$ ) and of charged amine $\left(-\mathrm{NH}_{3}{ }^{+}\right)$of Asp (7.5-8.0 ppm), to the two types of the interfacial carbonates. A partially resolved crosspeak appears at $\sim 4.9$ ppm which is suggestive of water molecules whose proximity to the interfacial carbonates suggests they are integral to the interface. Finally, a small crosspeak between the $\sim 10 \mathrm{ppm}$ hydrogen and the interfacial carbonates exposes bicarbonate species. The proximity of all hydrogen species of the amino acid to the interfacial carbonates is consistent with sparse molecular dispersion of occluded amino acids. 


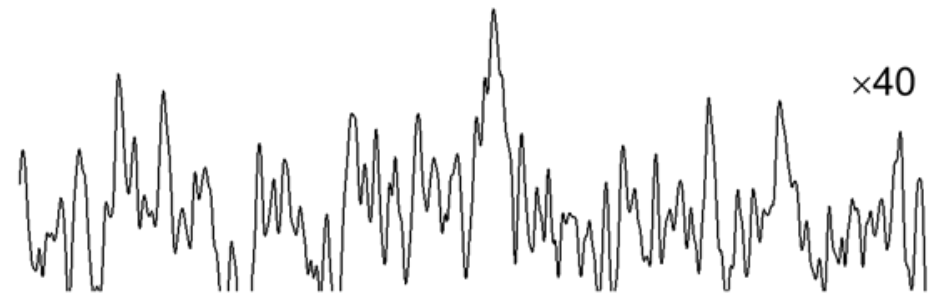

[Asp]-free calcite

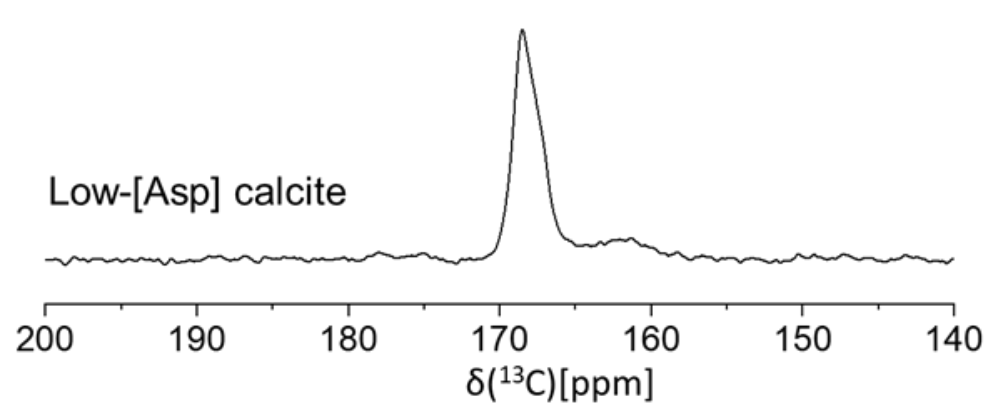

Figure S6: Normalized $75.4 \mathrm{MHz}{ }^{13} \mathrm{C}$ CPMAS NMR spectra of low-[Asp] calcite (bottom) and Asp-free calcite (middle) precipitated under the same conditions without Asp in the precipitation solutions. Top trace is a 40-fold vertical expansion of the CP spectrum of the Asp-free calcite sample, indicating the intensity of the CP-able carbonate peak is no more than $2.5 \%$ of that found in the low-[Asp] calcite.

Contact time employed for the two samples was 2ms; 2048 and 8192 transients were recorded for the low-[Asp] and Asp-free samples, respectively. 


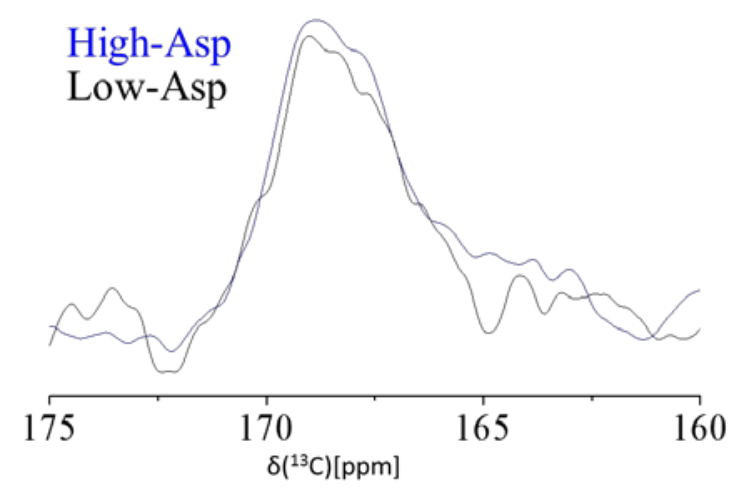

Figure S7: $75.4 \mathrm{MHz}{ }^{13} \mathrm{C}\left\{{ }^{15} \mathrm{~N}\right\} \mathrm{CP} \Delta \mathrm{S}$ REDOR of the Low-Asp (black) and the HighAsp (blue) vaterite coprecipitates (12.8 ms dipolar evolution; $\left.64 T_{\mathrm{R}}\right)$. These superimposed REDOR spectra show that the same chemical environments are formed, independent of Asp concentration in solution (10-fold) and Asp occlusion level ( 2fold).

Table S1: Association number calculations:

Experimental $\Delta S / S_{0}{ }^{15} \mathrm{~N}\left\{{ }^{13} \mathrm{C}\right\}$ CP-REDOR with dipolar evolution of $12.8 \mathrm{~ms}\left(64 T_{\mathrm{R}}\right)$, and calculated $\Delta S / S_{0}$ for ${ }^{15} \mathrm{~N} \cdot{ }^{13} \mathrm{C}_{n}$ with association numbers $n=2,3,4$. In these ${ }^{15} \mathrm{~N} \cdots{ }^{13} \mathrm{C}_{n}$ simulations the average $\Delta S / S_{0}$ value obtained from the ${ }^{13} \mathrm{C}\left\{{ }^{15} \mathrm{~N}\right\}$ REDOR measurements was employed. The analysis assumed isolated nuclei pair model ${ }^{59,60}$ which is justified by the low enrichment level, $10 \%-{ }^{13} \mathrm{C}$ carbonate. The resulting $\Delta S / S_{0}$ values (three right columns) which are closest to the experimentally measured values are in bold. to account for contributions from natural abundance ${ }^{13} \mathrm{C}$ 's, $5 \%$ were added to each calculated ${ }^{15} \mathrm{~N}\left\{{ }^{13} \mathrm{C}\right\} \Delta S / S_{0}$ value.

\begin{tabular}{|c|c|c|c|c|}
\hline \multirow[t]{3}{*}{ Coprecipitate } & \multicolumn{4}{|c|}{${ }^{15} N\left\{{ }^{13} C\right\} C P-R E D O R$} \\
\hline & \multirow{2}{*}{$\begin{array}{l}\Delta S / S_{0} \\
{[\%]}\end{array}$} & \multicolumn{3}{|c|}{ Calculated $\Delta S / S_{0}[\%]$} \\
\hline & & $\mathrm{N} \cdots \mathrm{C}_{2}$ & $\mathrm{~N} \cdots \mathrm{C}_{3}$ & $\mathrm{~N} \cdots \mathrm{C}_{4}$ \\
\hline High-[Asp] vaterite & $20 \pm 5$ & 15 & 20 & 25 \\
\hline Low-[Asp] intermediate & $22 \pm 5$ & 15 & 20 & 25 \\
\hline Low-[Asp] calcite & $25 \pm 7$ & 15 & 20 & 25 \\
\hline
\end{tabular}




\section{A computer model of a randomly doped cubic lattice}

Our model builds up a cubic lattice of dimension $N^{3}$ where a single "bulk" formula unit $\left(\mathrm{CaCO}_{3}\right)$ is represented by a cubic lattice unit (shown as white below); the larger Asp molecules are represented by two adjacent such lattice cubes (shown in red). We justify this oversimplified picture by realizing that there is a convenient relationship between the volumes of the Asp molecule and those of the carbonate lattices. The bulk densities of L-Asp, vaterite and calcite lead to formula unit volumes of $130 \AA^{3}, 63 \AA^{3}$ and $61 \AA^{3}$. For our purposes this suggests that choosing a "lattice constant" of $4 \AA$ edge gives a realistic approximation, where in any given of the $N^{3}$ cubes of our lattice are found either one formula unit of $\mathrm{CaCO}_{3}$, or one-half a formula unit of Asp.

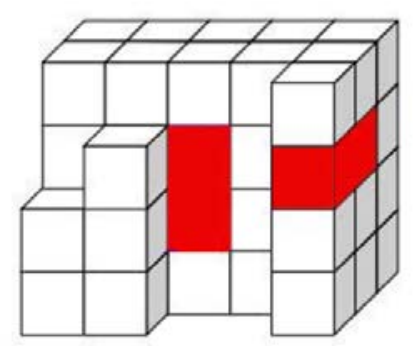

Scheme S1: Illustration of the cubic $\mathrm{CaCO}_{3}$ lattice doped with $\mathrm{L}-\mathrm{Asp}$. $\mathrm{A} \mathrm{CaCO}_{3}$ formula unit is represented by a white cube (of $4 \AA$ edge) while an occluded Asp is represented by two connected red cubes.

As Asp is incorporated into the growing carbonate lattice (as $\mathrm{Asp}^{1-}$ ), the lattice maintains electrical neutrality only where we remove four $\mathrm{CO}_{3}{ }^{2-}$ units and three $\mathrm{Ca}^{2+}$ units for each pair of inserted $\mathrm{Asp}^{1-}$. So as to mimic the inhibition observed in experiments, we have generated such lattices constrained such that no two Asp sites can be allowed to occupy adjacent lattice points, though in all other respects the red cubes are inserted randomly into the $N^{3}$ lattice, until a predetermined concentration ranging from $0.1-2.5 \%$ (containing our experimentally observed values of 0.5 and $1.2 \%)$.

Once a lattice is constructed, red-red and red-white nearest neighbor distances are tabulated subject to periodic boundary conditions, and repeated for increasing $N$ values until convergence is achieved; at the convergence volume $N^{3}$ the simulation is repeated multiple times so as to arrive at statistically significant results. Once lattices with converging statistical properties are constructed, nearest-neighbor distance distribution were tabulated. While the actual crystallographic lattice is, of course, not cubic, the cubes will typically contain a single $\mathrm{CaCO}_{3}$ formula unit-and reasonable approximate course-grained nearest-neighbor distances. 